\title{
La protesi totale oggi
}

\author{
Roberto D'Anchise \\ Responsabile U.O., di Chirurgia del Ginocchio I, Istituto Ortopedico Galeazzi IRCCS, Milano, Italia \\ aroberto.danchise@danchise.it
}

Pubblicato online: 29 ottobre 2013

(c) Springer-Verlag Italia 2013

Quando mi è stato chiesto dall'OTODI di curare un numero de Lo Scalpello sulle protesi di ginocchio ho accettato con piacere ed entusiasmo. Poi, ripensandoci, mi sono chiesto se non sarebbe stato l'ennesimo ripetitivo lavoro su questo argomento. Mi sono messo allora a considerare le mie esperienze passate sulle protesi e mi è venuto in mente un episodio che mi piace ricordare in questa occasione. Erano i primi anni Ottanta e mi ero interessato alle protesi di Freeman. Dopo aver trascorso un periodo a Londra presso il suo ospedale, iniziai a utilizzare questi impianti con risultati onestamente insoddisfacenti. Allora raccolsi gli esami radiografici dei miei insuccessi e ritornai da Freeman, chiedendogli in cosa avessi sbagliato. Lui, candidamente, mi rispose che non era colpa mia, ma sua, poiché la sua protesi non era adatta ai miei pazienti.

Ripensando a questo episodio ho riflettuto e ho considerato come progressivamente, negli anni, le tecniche si siano modificate, rendendo questo intervento estremamente affidabile e soprattutto riproducibile. Ho considerato inoltre come questa evoluzione abbia fatto sì che, fino al 2000, noi tutti fossimo convinti di aver raggiunto un ottimo livello di standardizzazione per le protesi, anche tenendo conto degli ottimi risultati nel tempo. Progressivamente, analizzando gli insuccessi, ci siamo resi conto che esisteva un ulteriore margine di miglioramento. Una piccola rivoluzione è nata con il concetto di "mini-invasività", che ci ha fatto capire come si potessero ottenere migliori risultati riducendo l'aggressione chirurgica, non tanto per quanto riguarda l'incisione, ma piuttosto per quanto riguarda muscoli e strutture articolari. Anche l'analisi delle protesi dolorose ci ha aiutato a capire molti aspetti, inducendoci a valutare alcuni concetti in parte trascurati in passato.

Gli ultimi 10 anni, quindi, hanno portato un notevole miglioramento sotto questo aspetto. Grazie alla nostra attenzione a particolari in passato ritenuti poco rilevanti, oggi possiamo dire che le nostre tecniche sono estremamente accurate, con risultati, anche immediati, molto soddisfacenti. Tutto questo mi ha fatto comprendere come, in realtà, fosse utile un aggiornamento sulle novità in campo protesico e mi ha spinto a programmare questo numero de Lo Scalpello chiedendo agli Autori di aiutarci a capire che cosa c'è "di nuovo". Ho rivolto l'invito a quelli che considero tra i migliori chirurghi protesici in Italia e, devo dire con un po' di sorpresa (considerando gli impegni sia professionali sia congressuali), ho ricevuto immediato ed entusiastico riscontro.

Sono certo che il risultato finale sarà estremamente positivo e utile per tutti coloro che affrontano queste problematiche. Un ringraziamento all'O.T.O.D.I., in particolare agli amici responsabili di questa rivista per la stima dimostrata nei miei confronti e a Springer-Verlag Italia, in particolare alla Dottoressa Paola Gregori, per il notevole impegno e supporto nella realizzazione di questo numero de Lo Scalpello.

CONFLITTO DI INTERESSE L'Autore dichiara una collaborazione con DePuy Johnson \& Johnson.

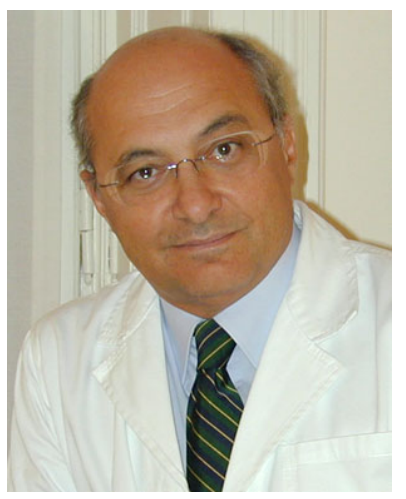

Roberto D'Anchise 